\title{
AN ISSUE TO THE COSMOLOGICAL CONSTANT PROBLEM
}

\author{
R TRIAY* \\ Centre de Physique Théorique * \\ CNRS Luminy Case 907, 13288 Marseille Cedex 9, France \\ triay@cpt.univ-mrs.fr
}

\begin{abstract}
On geometrical grounds, the cosmological constant problem turns out to be an artifact due to the unfounded link of this fundamental constant to vacuum energy density of quantum fluctuations.

Keywords: Cosmology; Vaccum Energy ; Cosmological Constant.
\end{abstract}

\section{Introduction}

The cosmological constant problem (CCP) is an enigma of modern cosmology among the particles physics and cosmology communities. The most comprehensive contribution to this problem can be found in. ${ }^{29}$ It is a matter of fact that its origin is intimately related to the status of the cosmological constant $\Lambda$. It was first assumed as a free parameter in the field equations with the aim of accounting for a static Universe and then rejected because a cosmological expansion was observed subsequently. Such an issue to the cosmological problem has provided us with (authority and/or simplicity) arguments in favor of $\Lambda=0$ until acceleration of the cosmological expansion could not be avoided for the interpretation of recent cosmological data. However, the related estimate does not agree by hundred orders of magnitude with its expected value as obtained from quantum field theories ${ }^{5,20,40}$ by interpreting $\Lambda / 8 \pi \mathrm{G}$ as vacuum energy density of quantum fluctuations. The aim of the present contribution is to understand this problem on geometrical grounds.

*Unité Mixte de Recherche (UMR 6207) du CNRS, et des universités Aix-Marseille I, Aix-Marseille II et du Sud Toulon-Var. Laboratoire affilié à la FRUMAM (FR 2291). 


\section{Status of the cosmological constant}

The reason why the status of the cosmological constant $\Lambda$ has long been discussed $^{6,11,30,38}$ has an historical origin, which still contributes to a sociological debate at the present time. The key point is that either $\Lambda$ is an universal constant, ${ }^{35}$ as it is clearly established in General Relativity (GR), as similarly as Newton constant of gravitation G, or it is associated to a particles/fields contribution to gravitation.

\subsection{Historical status of $\Lambda$}

For solving the cosmological problem, Einstein's goal was to obtain the gravitational field of a static universe, as it was supposed to be at that epoch. Similarly to the necessary modification of Poisson's equation for describing a uniform static distribution of dust in Newtonian gravity ${ }^{\mathrm{a}}, \Lambda$ was assumed in the gravitational field equations accordingly to GR. With Mach's principle in mind (origin of inertia), a consistent cosmological solution describing a spatially closed universe ${ }^{9}$ was derived, named as Einstein's model. A decade later, Friedmann's model ${ }^{14}$ was used by G. Lemaître ${ }^{18}$ for pointing out the cosmological expansion from Hubble's law, ${ }^{16}$ when Einstein's model was shown to be unstable ${ }^{8 b}$. This summarises very briefly the state of the art as recorded in contemporary textbooks. ${ }^{43}$ Henceforth, Friedmann's model with $\Lambda=0$ was preferred because of Einstein's definite renouncement from the point of view of "logical economy", ${ }^{10}$ what became the Standard world model. ${ }^{48}$ His confession $^{c}$ to G. Gamov ${ }^{15}$ stands probably for the historical reason why $\Lambda$ was wrongly understood as a free parameter in GR theory of gravitation by the majority of observational cosmologists until recently. Although, $\Lambda \neq 0$ has been unsuccessfully envisaged in the 60's for explaining observations. ${ }^{17,22,23,28,33}$ Nowadays, it is generally believed that $\Lambda \sim 2 h^{2} 10^{-56} \mathrm{~cm}^{-2}$ (where $h=H_{\circ} / 100 \mathrm{~km} \mathrm{~s}^{-1} \mathrm{Mpc}^{-1}$ ) is required for interpreting the CMB temperature fluctuations ${ }^{1,19,32,34}$ and for accounting of Hubble diagram of SN. ${ }^{24-26,31}$ Such a necessity was evident $^{\mathrm{d}}$ two decades earlier from statistical investigations based on quasars

\footnotetext{
a Such an approach has been used by Neumann (1896) in Newtonian theory, see R.C. Tolman. 43

${ }^{\mathrm{b}}$ i.e. in addition of suffering from a fine tuning problem on the values of $\Lambda$ and the specific density of energy of gravitational sources, any irregularity in their distribution causes either a collapse or an expansion.

"loc. cit. : "the biggest blunder of my life".

dit was ignored because not representative of the general consensus at that epoch, what is typical of the present days scholastic attitude.
} 
and galaxiess. ${ }^{2,2,3,12,13,41,44}$

\subsection{Geometrical status of $\Lambda$}

On geometrical grounds, Principle of General Relativity applied to gravity provides us with a unique interpretation of $\Lambda$. The gravitational field and its sources are characterized respectively by the metric tensor $g_{\mu \nu}$ on the space-time manifold $V_{4}$ and by a vanishing divergence stress-energy tensor $T_{\mu \nu}$. The gravitational field equations must be invariant with respect to the action of diffeomorphism group of $V_{4},{ }^{35,36}$ and therefore they read

$$
T_{\mu \nu}=-A_{0} F_{\mu \nu}^{(0)}+A_{1} F_{\mu \nu}^{(1)}+A_{2} F_{\mu \nu}^{(2)}+\ldots
$$

where $F_{\mu \nu}^{(n)}$ stands for a covariant tensor of degree $2 n$, defined by means of metric tensor $g_{\mu \nu}$ and its derivatives, and $A_{n}$ for a coupling constant, its value is estimated from observations. The $n=0,1$ order terms are uniquely defined

$$
F_{\mu \nu}^{(0)}=g_{\mu \nu}, \quad F_{\mu \nu}^{(1)}=S_{\mu \nu}=R_{\mu \nu}-\frac{1}{2} R g_{\mu \nu}
$$

where $R_{\mu \nu}$ is the Ricci tensor and $R$ the scalar curvature, whereas $F_{\mu \nu}^{n \geq 2}$ must be derived from additional principles.

Schwarzschild solution of Eq. (11) enables us to identify $A_{n=0,1}$ with Newton approximation, what provides us with modified Poisson equation ${ }^{35}$

$$
\operatorname{div} \tilde{\mathrm{g}}=-4 \pi \mathrm{G} \rho+\Lambda
$$

where $\vec{g}$ stands for the gravitational acceleration field due to sources defined by a specific density $\rho$, and the following identification of constants

$$
\mathrm{G}=\frac{1}{8 \pi A_{1}}, \quad \Lambda=\frac{A_{0}}{A_{1}}
$$

which shows their common status of universal constant.

\section{Modeling gravitational structures}

The space-time geometry is constrained by the presence of gravitational sources by means of tensor $T_{\mu \nu}$ in Eq. (1), each right hand terms contributes for describing the geometry within its effective scale. A dimensional analysis of field equations provides us with an estimation of their corresponding magnitudes, what is useful for modelling gravitational structures. Moreover, Newtonian approximation of gravitational field at large scale provides us with a simple way for interpreting $\Lambda$ effect. 


\subsection{Dimensional analysis}

According to GR, the speed of the light $c=1$ (i.e. time can be measured in unit of length ${ }^{\mathrm{e}} 1 \mathrm{~s}=2.99979245810^{10} \mathrm{~cm}$ ) and then $\mathrm{G}=7.4243 \times$ $10^{-29} \mathrm{~cm} \mathrm{~g}^{-1}$. Let us choose units of mass and of length ${ }^{\mathrm{f}}$, herein denoted respectively by $M$ and $L$. The correct dimensional analysis of GR sets the covariant metric tensor to have the dimension $\left[g_{\mu \nu}\right]=L^{2}$, and thus $\left[g^{\mu \nu}\right]=L^{-2},\left[R_{\mu \nu}\right]=1$ and $[R]=L^{-2}$. Since the specific mass density and the pressure belong to $T_{\nu}^{\mu}$, one has $\left[T_{\mu \nu}\right]=M L^{-1}$. Hence, according to Eq. (1), the dimensions of $A_{n}$ are the following

$$
\left[A_{0}\right]=M L^{-3}, \quad\left[A_{1}\right]=M L^{-1}, \quad \ldots\left[A_{n}\right]=M L^{2 n-3}
$$

which shows their relative contributions for describing the gravitational field with respect to scale. Namely, the larger their degree $n$ the smaller their effective scaleg. Equivalently, the estimation of $A_{0}$ demands observational data located at scale larger than the one for $A_{1}, e t c \ldots$ This is the reason why the $\Lambda$ effect is not discernible at small scale but requires cosmological distances.

\subsection{Newtonian gravity up to cosmological scales}

The observations show that gravitational structures within scales of order of solar system can be described by limiting the expansion solely to Einstein tensor $S_{\mu \nu}$, when cosmology requires also the first term. The transition scale between $A_{0}$ and $A_{1}$ is of order of $1 / \sqrt{\Lambda} \sim 7 h^{-1}$ Gyr. Although GR is preferred for investigating the dynamics of cosmic structures, Newton approximation given in Eq. (3) provides us with an easier schema for realizing the $\Lambda$ effect. Hence, the acceleration field due to gravity around a point mass $m$ reads

$$
\vec{g}=\left(-\mathrm{G} \frac{m}{r^{3}}+\frac{\Lambda}{3}\right) \vec{r}
$$

Since $\Lambda>0$, the gravity force is attractive at distance $r<r_{\circ}$ and repulsive at $r>r_{\circ}$ with a critical distance

$$
r_{\circ}=\sqrt[3]{3 m \mathrm{G} / \Lambda}
$$

where the gravity vanishes.

\footnotetext{
e This is the reason why any statement on the variation of $c$ is meaningless in GR. ${ }^{\mathrm{f}}$ Only two fundamental units can be chosen, the third one is derived.

g In other words, the contribution of $A_{0}$ dominates at scale larger than the one of $A_{1}$, etc...
} 


\section{The cosmological constant problem}

Let us assume :

(1) quantum fluctuations are sources of the gravitational field by means of the following stress-energy tensor ${ }^{\mathrm{h}}$

$$
T_{\mu \nu}^{\mathrm{vac}}=\rho_{\mathrm{vac}} g_{\mu \nu}, \quad \rho_{\mathrm{vac}}=\hbar k_{\max }
$$

in the field equations Eq.(1), where $k_{\max }$ stands for the ultraviolet momentum cutoff up to which the quantum field theory is valid, ${ }^{5}$ one has

$$
\rho_{\mathrm{vac}}^{E W} \sim 210^{-4}, \quad \rho_{\mathrm{vac}}^{Q C D} \sim 1.610^{15}, \quad \rho_{\mathrm{vac}}^{P l} \sim 210^{89}
$$

in units of $\mathrm{g} \mathrm{cm}^{-3}$. The reason why such an estimation is not unique comes from the perturbative aspect of the theory for describing the quantum world, what can be understood as a weakness of this approach.

(2) the cosmological term interprets as the contribution of quantum fluctuations

$$
\rho_{\Lambda}=\frac{\Lambda}{8 \pi \mathrm{G}}
$$

The difficulty of above hypotheses is that $\rho_{\text {vac }}$ differs from

$$
\rho_{\Lambda} \sim h^{2} 10^{-29} \mathrm{~g} \mathrm{~cm}^{-3}
$$

as obtained from astronomical observations, by 25-118 orders of magnitude. Such an enigma is named the cosmological constant problem. Other estimations of this quantum effect from the viewpoint of standard Casimir energy calculation scheme ${ }^{49}$ provide us with discrepancies of $\sim 37$ orders of magnitude. ${ }^{7}$ A similar problem happens when

$$
\Lambda_{\mathrm{vac}}=8 \pi \mathrm{G} \rho_{\mathrm{vac}}
$$

is interpreted as a cosmological constant. Indeed, if the quantum field theory which provides us with an estimate of $\rho_{\mathrm{vac}}$ is correct then the distance from which the gravity becomes repulsive in the sun neighborhood ranges from $r_{\circ}^{E W} \sim 210^{-2} h^{-2 / 3}$ a.u. down to $r_{\circ}^{P l} \sim 310^{-11} h^{-2 / 3} \AA$ depending on the quantum field theory, see Eq. (7). Obviously, such results are not consistent with the observations.

${ }^{\mathrm{h}}$ The usual picture which describes the vacuum as an isotropic and homogenous distribution of gravitational sources with energy density $\rho_{\mathrm{vac}}$ and pressure $p_{\mathrm{vac}}=-\rho_{\mathrm{vac}}$ (although this is not an equation of state) is not clear and not necessary for the discussion. 
Another version of the cosmological constant problem points out a fine tuning problem. It consists on arguing on the smallness of $\Lambda=\Lambda_{\mathrm{vac}}+\Lambda_{\circ}$, interpreted as an effective cosmological constant, where $\Lambda_{\circ}$ stands for a bare cosmological constant.

\subsection{Understanding the recession of galaxies}

The observations show that the dynamics of the cosmological expansion after decoupling era agrees with Friedmann-Lemaître-Gamov solution. It describes an uniform distribution of pressureless matter and CMB radiation with a black-body spectra, the field equations are given by Eq. (1) with $n \leq 1$. The present values of related densities are $\rho_{\mathrm{m}}=3 h^{2} 10^{-30} \mathrm{~g} \mathrm{~cm}^{-3}$ (dark matter included) and $\rho_{\mathrm{r}} \sim 5 h^{2} 10^{-34} \mathrm{~g} \mathrm{~cm}^{-3}$. Their comparison to the expected vacuum energy density $\rho_{\text {vac }}$ shows that if quantum fluctuations intervene in the dynamics of the cosmological expansion then their contribution prevails over the other sources (by 26-119 orders of magnitude today). Such an hypothesis provides us with a vacuum dominate cosmological expansion since primordial epochs. Therefore, one might ask whether such disagreements with observations can be removed by taking into account higher order $n \geq 2$ terms in Eq. (1). With this in mind, let us describe the dynamics of structures at scales where gravitational repulsion $(\Lambda>0)$ is observed. Since the values of universal constants $G$ and $\Lambda$ are provided by observations, it is more convenient to use adapted units of time $l_{g}$ and of mass $m_{g}$ defined as follows

$$
l_{g}=1 / \sqrt{\Lambda} \sim h^{-1} 10^{28} \mathrm{~cm}, \quad m_{g}=1 /(8 \pi G \sqrt{\Lambda}) \sim 4 h^{-1} 10^{54} \mathrm{~g}
$$

herein called gravitational units. They are defined such that the field equations read in a normalized form

$$
T_{\mu \nu}=-g_{\mu \nu}+S_{\mu \nu}+A_{2} F_{\mu \nu}^{(2)}+\ldots
$$

i.e. $A_{0}=A_{1}=1$, where the stress-energy tensor $T_{\mu \nu}$ accounts for the distribution of gravitational sources. It is important to note that, with gravitational units, Planck constant reads

$$
\hbar \sim 10^{-120}
$$

Indeed, such a tiny value as quantum action unit compared to $\hbar=1$ when quantum units are used instead, shows clearly that Eq. (14) truncated at order $n \leq 1$ is not adapted for describing quantum physics. ${ }^{45,46}$ This is the main reason why it is hopeless to give a quantum status to $\Lambda .{ }^{41}$ As 
approximation, because of dimensional analysis described above, the contribution of higher order terms being the more significant as the density is large, Eq. (14) can be splited up with respect to scale into two equations systems. The first one corresponds to terms of order $n<2$ (the usual Einstein equation with $\Lambda$ ) and the second one

$$
T_{\mu \nu}^{\mathrm{vac}}=A_{2} F_{\mu \nu}^{(2)}+\ldots
$$

stands for the field equations describing the effect of quantum fluctuations on the gravitational field at an appropriated scale (quantum), interpreted as correction of the RW metric $g_{\mu \nu}$. The identification of constants $A_{n}$ (e.g., $A_{2}=\hbar$ ) and the derivation of tensors $F_{\mu \nu}^{(n)}$ with $n \geq 2$ requires to model gravitational phenomena at quantum scale, see e.g. ${ }^{39,42}$ Unfortunately, the state of the art does not allow yet to provide us with a definite answer for defining the right hand term of Eq. (16), see e.g. ${ }^{27}$

\section{Conclusion}

Seeking for the contribution of quantum fluctuations into the cosmological constant is a motivation that inherits from the previous attitude consisting on rejecting the cosmological term. On the other hand, to rescale the field equations for describing the cosmological expansion prevents us to assume the vacuum acting as a cosmological constant. As a consequence, one understands that such an interpretation turns to be the origin of the cosmological constant problem. In other words, such a problem is the price to pay for identifying $\Lambda_{\text {vac }}$ to the cosmological constant. Because the understanding of quantum gravity is still an ongoing challenge, the correct field equations describing the contribution to gravity of quantum fluctuations are not yet established. However, the dimensional analysis shows that the related gravitational effects are expected at small (quantum) scales and do not participate to the general expansion of the universe according to observations.

\section{References}

1. A. Benoit et al., Astron. Astrophys., 399, L25 (2003)

2. G. Bigot, H.H.Fliche, R. Triay, Astron. Astrophys., 206, 1 (1988)

3. G. Bigot, R. Triay, in The quest for the fundamental constants in Cosmology, $9^{\text {th }}$ Moriond Astrophysics meeting, eds J. Audouze and J. Trân Thanh Vân, Editions Frontières (1989)

4. S.M. Carroll, W.H. Press, E.L. Turner, Annu. Rev. Astron. Astrophys. 30, 499 (1992) 
5. S.M. Carroll, Living Reviews in Relativity, 4,1 (2001)

6. J.C. Charlton, M.S. Turner, Astrophys. J., 313,495 (1987)

7. I.O. Cherednikov, Acta Phys. Pol. B, 33 (2002)

8. A.S. Eddington, Month. Not. Roy. Astron. Soc., 90, 668 (1930)

9. A. Einstein, Sitz. Preuss. Akad. wiss. Phys-Math, 142, 87 (1917)

10. A. Einstein, Sitz. Preuss. Akad. wiss. Phys-Math, 235,37 (1931); Appendix to the 2nd edn. of The Meaning of Relativity,(1945) on the "cosmological problem"; reprinted in (all later editions) Princeton University Press (1955), p.109

11. J.E. Felten, R. Isaacman Rev. Mod. Phys., 58,689 (1986)

12. H.H. Fliche, J.M. Souriau, Astron. \& Astrophys., 78, 87 (1979)

13. H.H. Fliche, J.M. Souriau, R. Triay, Astron. \& Astrophys., 108, 256 (1982)

14. A. Friedmann, Z. Phys., 10,377 (1922); ibid. 21,326 (1924)

15. G. Gamow, in My world line, Viking Press, New York (1970), p.44

16. E.P. Hubble, Proc. Nat. Acad. Sci., 15, 168 (1927)

17. N. Kardashev, Astrophys. J., 150, L135 (1967)

18. G. Lemaître, Ann. Soc. Sci. Bruxelles., 47 A, 49 (1927); Month. Not. Roy. Astron. Soc., 91, 483 (1931)

19. C.B. Netterfield et al., Astron. J., 571, 604 (2002)

20. T. Padmanabhan, Phys. Rep., 380,235 (2003)

21. J.V. Peach, Astrophys. J., 159, 753 (1970)

22. V. Petrosian, E.E., Salpeter, P. Szekeres Astrophys. J., 147, 1222 (1967)

23. V. Petrosian, E.E., Salpeter, Astrophys. J.., 151, 411 (1968)

24. J.V. Perlmutter et al., Nature, 391, 51 (1998)

25. J.V. Perlmutter et al., Astrophys. J., 483, 565 (1999)

26. A.J. Riess et al., Astron. J., 116, 1009 (1998)

27. C. Rovelli, Int. Jour. Mod. Phys. D, 12,1509 (2003)

28. M. Rowan-Robinson, Month. Not. Roy. Astron. Soc., 141, 445 (1968)

29. V. Sahni, A. Starobinsky, Int. Jour. Mod. Phys. D, 9, 373 (2000)

30. A. Sandage, Ann. Rev. Astron. Astrophys., 26, 561 (1988)

31. P.B. Schmidt et al., Astrophys. J., 507, 46 (1998)

32. J.L. Sievers et al., Astrophys. J., 591, 599 (2003)

33. I. Shklovsky, Astrophys. J., 150, L1 (1967)

34. D.N. Spergel et al.; Astrophys. J. Suppl., 148, 175 (2003)

35. J.M. Souriau, Géométrie et relativité (Hermann, Paris, 1964)

36. J.M. Souriau, Ann. Inst. H. Poincaré, XX (4), 315 (1974)

37. J.M. Souriau, in Géométrie symplectique et physique mathématique, Colloques Internationaux CNRS, 237, 59 (1974)

38. J.M. Souriau, in Journées Relativistes - 1976, ULB Bruxelles (1977)

39. K.S. Stelle, Phys. Rev. D, 16,953 (1977)

40. M. Straumann, in 1rst Sem. H. Poincaré. Paris (2002)

41. J.M. Souriau, R. Triay, Grav. \& Cosm., 3,(9),51 (1997)

42. G.'t Hooft, M. Veltmann, Ann. Inst. H. Poincaré, (A) Phys. Th. XX (1), 69 (1974)

43. R.C. Tolman, in Relativity Thermodynamics and Cosmology, Oxford at the Clarendon Press (1934), p.344 
44. R. Triay, in Large Scale Structures and Peculiar Motions in the Universe, eds. D.W. Latham and L.N. da Costa, ASP conference series (1989)

45. R. Triay, in The Cosmological Model, $37^{\text {th }}$ Moriond Astrophysics meeting, Les Arcs, Editions Frontière (2002)

46. R. Triay, in Proceedings of the Tenth Marcel Grossmann Meeting on General Relativity, p. 2266 (Rio de Janeiro, Brazil 2003) . Edit. M. Novello \& S.P. Bergliaffa, Ser. Edit. R. Ruffini. World Scientific (2006)

47. R. Triay, Int. Journ. Mod. Phys. D, 1410,1667 (2005)

48. S. Weinberg, Gravitation and Cosmology, Ed. John Wiley \& Sons Inc.

49. Ya. B. Zel'dovich, JETP Lett., 6, 316 (1967)

50. Ya. B. Zel'dovich, I.D. Novikov, in The Structure and Evolution of the Universe, Vol. 2, Ed. G. Steigman, The University of Chicago press (1983) 